Chronic lymphocytic leukemia (CLL) is the most commonly diagnosed type of leukemia in Western Europe and North America, and represents about $30 \%$ of all leukemias in adults. Chronic lymphocytic leukemia is a disease of the elderly, who are often in poorer general health and burdened with multiple comorbidities. These factors affect the decision making when choosing an appropriate method of treatment.

In recent years there has been significant progress in the treatment of chronic lymphocytic leukemia, first due to the introduction of immunochemotherapy with monoclonal antibodies and latterly small molecules, like tyrosine kinase inhibitors targeting B-cell receptor signaling. This article discusses the current diagnostic principles, the most important prognostic factors and therapeutic options, available in first-line treatment and in refractory/resistant disease, including high-risk CLL, both for patients with good and those with poor performance status. It also presents important novel molecules which have been evaluated in clinical trials.

Key words: chronic lymphocytic leukemia, clinical symptoms, diagnosis, prognostic factors, treatment.

Contemp Oncol (Pozn) 2015; 19 (5): 361-367 DOI: $10.5114 /$ wo.2015.55410

\section{Current concepts in diagnosis and treatment of chronic lymphocytic leukemia}

\author{
Iwona Hus ${ }^{1}$, Jacek Roliński²
}

${ }^{1}$ Department of Clinical Transplantology, Medical University of Lublin, Poland ${ }^{2}$ Chair and Department of Clinical Immunology, Medical University of Lublin, Poland

\section{Introduction}

Chronic lymphocytic leukemia (CLL) is the type of leukemia most commonly diagnosed in Western Europe and North America, with an incidence rate of 4.2/100,000 [1]. Chronic lymphocytic leukemia is a disease of the elderly; the median age at diagnosis is 67-72 years [2], and approximately $70 \%$ of patients are over 65 years old. The only known risk factor is a family history of this disease. For relatives of CLL patients, the risk increases by a factor of $2.5-7.5[3]$.

Onset of the disease is usually asymptomatic; only abnormalities in whole blood count such as leukocytosis with lymphocytosis are found. In more advanced stages, lymphadenopathy, hepatomegaly/splenomegaly, recurrent infections, weakness, pallor, hemorrhagic diathesis and general symptoms such as fever, weight loss and night sweats are observed. Nowadays, due to more frequent routine blood counts, more and more cases of CLL are diagnosed at an early, asymptomatic stage.

\section{Principles of chronic lymphocytic leukemia diagnosis}

The basic criterion for CLL diagnosis is an absolute number of peripheral blood B cells greater than $5.0 \mathrm{G} /$, lasting more than three months; clonality should be confirmed by flow cytometry ( $\kappa$ or $\lambda$ light chains) [4]. Chronic lymphocytic leukemia is characterized by a specific immunophenotype. In addition to the typical markers of B lymphocytes (CD19, CD20, CD23), 95\% of patients with leukemia exhibit the cell surface antigen CD5 [5, 6]. In most cases, the immunophenotyping of peripheral blood lymphocytes by flow cytometry allows the establishment of a diagnosis, and distinguishes CLL from other B-cell lymphomas. In the differential diagnosis, one should take into account other indolent lymphomas (marginal zone lymphoma, hairy cell leukemia) and mantle cell lymphoma (MCL). In cases of doubt, methods which are helpful include histopathology and cytogenetic evaluation (FISH - fluorescent in situ hybridization) (for example, t(11;14) translocation, typical of $\mathrm{MCL}$ ).

Bone marrow examination is not required to establish the diagnosis of CLL [4]. It is recommended in patients with cytopenias to differentiate between autoimmune cytopenias and the replacement of normal bone marrow cells by leukemic cells [4]. Bone marrow assessment should be performed before the start of therapy, especially myelosuppressive ones [1, 4, 7], and repeated in the case of persistent cytopenias after the treatment. Marrow biopsy is only recommended in clinical trials to confirm complete remission, but in practice it is optional and depends on the discretion of the physician [4].

Lymph node biopsy is indicated only in case of suspicion of transformation into a more aggressive type of lymphoma (Richter's syndrome). Tests performed at diagnosis of CLL are shown in Table 1. 
Table 1. Tests to establish the diagnosis of chronic lymphocytic leukemia [4]

$\begin{array}{ll}\text { Test } & \text { Indications } \\ \begin{array}{l}\text { Complete blood count and white blood } \\ \text { cells smear }\end{array} & \text { Always } \\ \begin{array}{l}\text { Immunophenotyping } \\ \text { of peripheral blood lymphocytes }\end{array} & \text { Always } \\ \begin{array}{l}\text { Bone marrow biopsy (aspiration, } \\ \text { trephine biopsy) }\end{array} & \begin{array}{l}\text { Not necessary for a diagnosis. Recommended only to differentiate autoimmune cytopenia } \\ \text { fymph node biopsy }\end{array} \\ & \begin{array}{l}\text { Not necessary for diagnosis. Recommended only in cases of suspected Richter's } \\ \text { transformation. }\end{array} \\ \text { Cytogenetic evaluation } & \begin{array}{l}\text { Only in the case of diagnostic problems, e.g., translocation of t(11:14), typical of mantle cell } \\ \text { lymphoma. }\end{array}\end{array}$

\section{The significance of prognostic factors in chronic lymphocytic leukemia}

A characteristic feature of CLL is its heterogeneous clinical course [8]. Some patients do not require treatment for many years, if ever, whilst for others treatment is necessary soon after diagnosis. Staging of the disease is based on the Binet classification system introduced in 1975 [9] and the Rai staging system presented in 1981 [10] (Table 2).

However, both systems have limited value in determining the clinical course of the disease in individual cases, and in the identification of progressive CLL, especially in the early stages of disease.

Apart from the stage of the disease, clinical parameters (age, sex, comorbidities) and laboratory parameters ( $\beta 2$-microglobulin, lactate dehydrogenase, thymidine kinase, soluble CD23 receptor, lymphocyte doubling time, the type of marrow infiltration by leukemic lymphocytes) have significant prognostic value [5, 11-16].

Over the past several years, a group of so-called new prognostic factors has been developed. This group in- cludes the mutational status of immunoglobulin heavy chain variable region (IGVH) genes [17-19], the expression of ZAP-70 protein in CLL cells [20-22], the expression of CD38 antigen on leukemic cells [23, 24] and cytogenetic abnormalities.

Cytogenetic abnormalities have the most important practical significance, especially deletions of $11 q$ and $17 p$ [25]. The presence of $11 \mathrm{q}$ deletion correlates with significant lymphadenopathy and rapid progression of the disease [26], while 17p deletion is associated with short survival time and lack of response to treatment with alkylating agents and purine analogs [25]. Results of the CLL8 trial showed that adding rituximab to FC (fludarabine and cyclophosphamide) chemotherapy makes the prognosis of patients with 11 q deletion similar to those without the deletion [27]. Unfortunately, this has no significant effect on improving the prognosis of patients with the $17 p$ deletion. The $17 p$ deletion occurs at diagnosis in about $5-7 \%$ of all CLL patients, and its frequency increases significantly with the progression of the disease. For patients in whom

Table 2. Chronic lymphocytic leukemia staging systems by Rai et al. [10] and Binet et al. [9]. Median survival time based on updated data [11]

\begin{tabular}{|c|c|c|}
\hline Stage & Parameter & Median overall survival \\
\hline Rai classification & & Modified Rai classification \\
\hline 0 & absolute lymphocytosis > 5 G/l & $>10$ years/low risk \\
\hline 1 & $\begin{array}{l}\text { absolute lymphocytosis > } 5 \mathrm{G} / \mathrm{l} \\
+ \text { lymphadenopathy }\end{array}$ & $>8$ years/intermediate risk \\
\hline II & $\begin{array}{l}\text { absolute lymphocytosis > } 5 \mathrm{G} / / \\
\text { + splenomegaly or hepatomegaly } \\
\text { +/- lymphadenopathy }\end{array}$ & $>8$ years/intermediate risk \\
\hline III & $\begin{array}{l}\text { absolute lymphocytosis > 5 G/l } \\
\text { + hemoglobin < } 11 \mathrm{~g} / \mathrm{l} \\
\text { +/- lymphadenopathy, } \\
\text { +/- splenomegaly or hepatomegaly }\end{array}$ & 6.5 years/high risk \\
\hline IV & $\begin{array}{l}\text { absolute lymphocytosis > 5 G/l } \\
+ \text { PLT < } 100 \mathrm{G} / \text { l } \\
\text { +/- lymphadenopathy, } \\
\text { +/- splenomegaly or hepatomegaly } \\
\text { +/- hemoglobin }<11 \mathrm{~g} / /\end{array}$ & 6.5 years/high risk \\
\hline \multicolumn{3}{|l|}{ Binet classification } \\
\hline A & involvement of $<3$ lymphoid tissue areas & $>10$ years \\
\hline B & involvement of $\geq 3$ lymphoid tissue areas & $>8$ years \\
\hline $\mathrm{C}$ & hemoglobin $<10 \mathrm{~g} / \mathrm{dl}$ and/or PLT $<100 \mathrm{G} / \mathrm{l}$ & 6.5 years \\
\hline
\end{tabular}


therapy overcoming the resistance may be applied, an assessment of the 17p deletion should be undertaken before each line of treatment, keeping in mind that the presence of 17p deletion alone without signs of CLL progression is not an indication for treatment.

The most recent prognostic factors, assessed by whole genome sequencing, include NOTCH1, SF3B1 and BIRC3 mutations. At present, determination of these mutations is not recommended in clinical practice.

\section{Initial clinical evaluation}

Initial clinical evaluation of a patient with diagnosis of CLL should include:

- detailed physical examination including lymph nodes, liver and spleen assessment,

- determination of the clinical stage (according to Rai or Binet classification),

- finding out the cause of cytopenia (autoimmune, bone marrow infiltration by leukemic cells, hypersplenism, other), if present at diagnosis.

Laboratory tests recommended at CLL diagnosis include [7]:

- whole blood count with white blood cell smear,

- reticulocyte count,

- direct antiglobulin test (DAT, Coombs test),

- routine biochemical assessment of renal and hepatic function,

- serum immunoglobulins concentration (IgG, IgA, IgM).

For patients with a normal total IgG level experiencing recurrent infections, consider an assessment of IgG subclasses IgG1, IgG2, IgG3, IgG4, if possible.

In clinical practice, there are no recommendations for computed tomography (CT) scanning in patients with early asymptomatic stages of CLL or for monitoring of patients after the treatment completion [7], while CT is necessary to assess the tumor burden as well as the response to the therapy in clinical trials. In routine practice, CT scanning may be indicated in patients treated with intensive chemoimmunotherapy [7]. Positron emission tomography (PET) is not applicable in patients with CLL, except in cases of Richter's transformation.

Patients should undergo the following tests before the start of intensive chemotherapy or immunotherapy:

- cytogenetic evaluation (17p and 11q deletions by FISH),

- virological tests: hepatitis B and C viruses (HBV, HCV), cytomegalovirus (CMV), human immunodeficiency virus (HIV).
The most serious complication of therapy with alemtuzumab is the reactivation of a cytomegalovirus infection.

Immunotherapy with rituximab and other anti-CD20 monoclonal antibodies might be associated with reactivation of HBV infection.

\section{Indications for treatment of chronic lymphocytic leukemia}

In most cases, establishing the diagnosis of CLL does not indicate the need for the initiation of therapy. Treatment is not recommended for patients with CLL in early stages. Only patients with active disease require therapy. Generally accepted indications for CLL treatment according to the IWCLL (International Workshop on Chronic Lymphocytic Leukemia) [4] are shown in Table 3. One has to remember that a high number of lymphocytes alone, without signs of leukostasis, should not be an indication to start treatment.

\section{Assessment of response to therapy}

The current criteria for the response to therapy (by IWCLL) were published by Hallek et al. in 2008 [4]. Complete remission (CR) requires the fulfillment of all of the following criteria, assessed at least two months after completion of the therapy: absence of lymphadenopathy (lymph node size $<1.5 \mathrm{~cm}$, evaluated in clinical trials, using a CT scan and in clinical practice, using a physical examination); the absence of hepato- and splenomegaly; peripheral blood lymphocyte count $<4000 / \mu$; t the percentage of lymphocytes in the bone marrow $<30 \%$, with normal cellularity, without B lymphocyte clusters; peripheral blood parameters: neutrophils > $1.5 \mathrm{G} / \mathrm{l}$, PLT count $>100 \mathrm{G} / \mathrm{l}$, Hgb $>11 \mathrm{~g} / \mathrm{dl}$.

In clinical trials, complete remission should be determined on the basis of CT scans and bone marrow assessment. According to recent recommendations [1], assessment of patients' response in clinical trials should include the assessment of MRD using four-color cytometry or ASO-PCR (allele-specific oligonucleotide polymerase chain reaction). Both complete and partial remission should be referred to as MDR+ or MDR-. Minimal residual disease assessment is not currently recommended in the clinical practice.

In the patients fulfilling the criteria of complete remission (as confirmed by bone marrow examination), with the persistence of anemia, thrombocytopenia or neutropenia (related to treatment toxicity), the response is defined as CR with incomplete marrow recovery [4].

Table 3. Indications for CLL treatment according to IWCLL [4]

Advanced clinical stage of the disease (Rai 3 or 4, Binet C)

A significant or progressive lymphadenopathy (longest dimension $>10 \mathrm{~cm}$ ) or splenomegaly $(>6 \mathrm{~cm}$ below the costal margin)

Cytopenia due to disease progression or autoimmune disorders (lack of response to corticosteroids or other standard treatments)

General symptoms (weight loss, fever, fatigue, infection)

Lymphocyte doubling time of $<6$ months, or an increase of $>50 \%$ in less than two months (for patients with lymphocytosis $<30 \mathrm{G} / \mathrm{l}$, should not be the only indication for treatment)

Richter's transformation 


\section{Patients' assignment to the appropriate treatment regimen}

The choice of an appropriate treatment for patients with CLL depends primarily on the expected tolerance of chemo-or immunochemotherapy, assessed on the basis of parameters such as:

- age,

- performance status according to ECOG (Eastern Cooperative Oncology Group) scale or Karnofsky index,

- comorbidities,

- creatinine clearance ( $\geq 70 \mathrm{ml} / \mathrm{min})$.

The decision on whether a patient can receive intensive treatment is in many cases difficult, particularly in elderly patients. In the assessment of comorbidities in patients with cancer, appropriate scales may be helpful, such as the CIRS (Cumulative Illness Rating Scale) used by the German CLL Study Group (GCLLSG) [28].

The method of treatment is also dependent on whether it is:

- first- or subsequent-line therapy,

- high-risk CLL, defined by the presence of 17p deletion/ TP53 mutation (in untreated and treated patients, with indications for treatment) or resistance/relapse within two years after immunochemotherapy [29].

\section{First-line treatment}

First-line treatment for patients in good general condition without significant comorbidities

The first-line treatment recommended for CLL patients in good general condition with no significant comorbidities, and without a 17p deletion, is immunochemotherapy with purine analogues (fludarabine or cladribine), cyclophosphamide and anti-CD20 monoclonal antibody - rituximab (FCR or CCR regimens). The results of CLL8 trail of the GCLLSG showed that FCR immunochemotherapy prolongs the overall survival in patients with CLL [27].

The results of the CLL3 trial, conducted by the PALG (Polish Adult Leukemia Study Group), demonstrated similar efficacy and safety of FC (fludarabine, cyclophosphamide) and CC (cladribine, cyclophosphamide) regimens in the first-line treatment of CLL [30]. According to the GCLLSG, eligibility criteria for FCR (fludarabine, cyclophosphamide, rituximab) immunochemotherapy are:

- creatinine clearance $\geq 70 \mathrm{ml} / \mathrm{min}$ and

- CIRS score $\leq 6$ [31].

Patients not fulfilling the above criteria should be treated using less toxic regimens.

It is recommended to give patients 6 cycles of FCR immunochemotherapy, as this increases the probability of the eradication of minimal residual disease (MRD), which is an independent predictor of longer progression-free survival (PFS) and longer overall survival (OS) [32]. It is necessary to carefully monitor any adverse effects, and a patient must exhibit good tolerance in order to complete six cycles of treatment. The results of CLL10 study of the GCLLSG showed no benefit in PFS in patients aged $\geq 65$ years treated with FCR over BR (bendamustine, rituximab) protocol [33]. Thus, taking into account higher hematologi- cal toxicity of FCR, BR protocol might be considered as first line therapy in fit, elderly patients with CLL.

\section{Patients in worse general condition, with comorbidities}

The drug used for many years in CLL treatment, and still recommended for elderly patients burdened with numerous comorbidities, is chlorambucil.

Advantages of using chlorambucil are the convenient oral route of administration, the relatively good patient tolerance

and the inexpensive cost of therapy. However, a complete response can be achieved only in $5-10 \%$ of patients.

Higher response rates and longer progression-free survival could be achieved when chlorambucil is combined with rituximab as it was demonstrated in two phase II clinical studies [34,35]. More recently, the results of multicenter randomized phase III study conducted by GCLLSG (CLL11) showed that immunochemotherapy with chlorambucil combined with rituximab or obinutuzumab (the new, type 2, glycoengineered anti-CD20 antibody) improves overall survival comparing to chlorambucil in CLL patients not qualified to FCR due to comorbidities or impaired renal function. Immunochemotherapy with obinutuzumab is more efficient in terms of higher responses rates, longer PFS and more frequent eradication of MRD as compared to rituximab and chlorambucil $[36,37]$. In COMPLEMENT-1 trail, ofatumumab (a type 1 humanized anti-CD20 antibody) combined with chlorambucil was also proved to be more effective than chlorambucil monotherapy, inducing higher response rates and longer PFS in patients with CLL inappropriate for fludarabine-based therapy due to advanced age and/or comorbidities [38]. Current ESMO [39] and NCCCN [40] guidelines recommend chlorambucil in combination with anti-CD20 antibodies (rituximab, obinutuzumab, ofatumumab) as a standard first line therapy in CLL patients that due to comorbidities are not candidates to intensive immunochemotherapy .

Other treatment options include bendamustine in monotherapy or in combination with rituximab (BR regimen) [41-43], FCR-Lite [44], Q-Lite [45] regimens or reduced doses of purine analogs [46].

\section{Treatment of relapsed disease}

Indications to initiate treatment in relapsed CLL are the same as for the first-line therapy. The choice of regimen for the second- and subsequent-line therapy depends, as in the case of first-line therapy, on the patient's performance status and comorbidities. The type of first-line therapy, its toxicity, and the duration of the response should also be taken into account. Regardless of the method of therapy, if a response lasting at least $24-36$ months was achieved, the regimen used in the first line should be repeated [2]. In patients with a good performance status, without the $17 p$ deletion/TP53 mutation, and for whom first-line treatment purine analogs or immunochemotherapy were used, the FCR/CCR or BR regimens should be used. Patients who received alkylating agents as a first-line treatment, and their general condition allows the use of intensive immu- 
nochemotherapy, may benefit from the FCR regimen (on the basis of the results of the REACH study) [47]. Recently two new small molecules, tyrosine kinase inhibitors (TKI) blocking B-cell receptor (BCR) signaling have been approved for the treatment of patients with CLL who received at least one prior therapy. Ibrutinib that is irreversible inhibitor of Bruton's kinase, was shown to be more effective than ofatumumab in the patients with relapsed CLL by inducing higher response rates as well as longer PFS and OS in the RESONATE trail [48]. Idelalisib is a selective and reversible inhibitor of phosphatidylinositide 3-kinase (PI3K). In patients with relapsed CLL, idelalisib in combination with rituximab was proved to induce higher response rates and longer both PFS and OS comparing to rituximab monotherapy [49]. Both ibrutinib and idelalisib are well tolerated in long-term therapy and they could be used in elderly patients with coexistent conditions. Alternatives for less fit patients are: BR [50], FCR-lite, corticosteroids at high doses (high dose methylprednisolone, HDMP) +/-rituximab [51] or rituximab, cyclophosphamide and dexamethasone (the RCD regimen); the latter is especially recommended for patients with autoimmune cytopenias $[52,53]$. Patients with relapsed CLL are encouraged to participate in clinical trials, if possible.

\section{Treatment of high-risk chronic lymphocytic leukemia}

$B C R$ inhibitors are considered nowadays the most effective conventional treatment for CLL patients with $17 p$ deletion/TP53 mutation however their efficacy is still inferior comparing to the patients without these abnormalities. Ibrutinib and idelalisib + rituximab are recommended treatment for the CLL patients with 17p deletion/TP53 mutation both in first, as well as in the subsequent lines of therapy [39]. Also in patients with refractory CLL or with relapse within 24-36 months from the start of initial therapy BCR inhibitors are recommended [39]. An alternative for the high risk patients is alemtuzumab $+/$ - corticosteroids [54], or fludarabine (FluCam) [55]. Nowadays, alemtuzum$a b$ is only available within a compassionate use program of Sanofi. In patients with a good performance status responding to the therapy, allogeneic hematopoietic cell transplantation (alloHSCT) may be considered. The indications for and timing of alloHSCT are under discussion after the introduction of small molecules into CLL therapy. This procedure needs to be taken under consideration in fit CLL patients failing to several lines of therapy or in patients with high-risk CLL relapsing to one TKI and respond to the second regimen [39]. In less fit patients with relapse within 24 - 36 months after immunochemotherapy, with no del17p or TP53 mutation, protocols like BR or FCR-lite could be used. Also corticosteroids in high doses combined with rituximab might be considered in high-risk disease $[56,57]$. An improvement of the prognosis in patients with high-risk CLL still remains an unmet need, so patients are encouraged to participate in clinical trials with new molecules, such as BCL-2 antagonist, ABT-199 (venetoclax) that shows efficacy in patients with high-risk CLL (refractory to fludarabine or with 17p deletion) [58] or new BCR inhibitors or immunomodulatory agents.
The authors thank Mundipharma Polska Sp. z o.o., Warsaw,

Poland, for sponsoring this paper. Conflict of interest: Iwona Hus - honoraria, travel grants from Roche, Janssen, Gilead, Mundipharma; Jacek Rolinski declares no conflict of interest. Technical support and language assistance were provided by Proper Medical Writing, Warsaw, Poland.

\section{References}

1. Eichhorst B, Dreyling M, Robak T, Montserrat E, Hallek M. ESMO Guidelines Working Group. Ann Oncol 2011; 22 Suppl 6: vi50-4.

2. Hallek M. Chronic lymphocytic leukemia: 2013 update on diagnosis, risk stratification and treatment. Am J Hematol 2013; 88: 803-16.

3. Slager SL, Kay NE. Familial chronic lymphocytic leukemia: what does it mean to me? Clin Lymphoma Myeloma 2009; 9 Suppl 3: S194-7.

4. Hallek M, Cheson BD, Catovsky D, et al. International Workshop on Chronic Lymphocytic Leukemia. Guidelines for the diagnosis and treatment of chronic lymphocytic leukemia: a report from the International Workshop on Chronic Lymphocytic Leukemia updating the National Cancer Institute-Working Group 1996 guidelines. Blood 2008; 111: 5446-56.

5. Rozman C, Montserrat E. Chronic lymphocytic leukemia. N Engl J Med 1995; 333: 1052-7.

6. Jovanovic D, Djurdjevic P, Andjelkovic N, Zivic L. Possible role of CD22, CD79b and CD20 expression in distinguishing small lymphocytic lymphoma from chronic lymphocytic leukemia. Contemp Oncol (Pozn) 2014; 18: 29-33.

7. Oscier D, Dearden C, Eren E, et al. British Committee for Standards in Haematology. Guidelines on the diagnosis, investigation and management of chronic lymphocytic leukaemia. Br J Haematol 2012; 159: 541-64.

8. Caligaris-Cappio F, Hamblin TJ. B-cell chronic lymphocytic leukemia: a bird of a different feather. J Clin Oncol 1999; 17: 399-408.

9. Binet JL, Auquier A, Dighiero G, et al. A new prognostic classification of chronic lymphocytic leukemia derived from a multivariate survival analysis. Cancer 1981; 48: 198-206.

10. Rai KR, Sawitsky A, Cronkite EP, Chanana AD, Levy RN, Pasternack BS. Clinical staging of chronic lymphocytic leukemia. Blood 1975; 46: 219-34.

11. Wierda WG, O’Brien S, Wang X, et al. Characteristics associated with important clinical end points in patients with chronic lymphocytic leukemia at initial treatment. J Clin Oncol 2009; 27: 1637 43.

12. Berrebi A, Bassous L, Haran M, Shtalrid M, Shvidel L. The significance of elevated beta 2-microglobulin (b2-m) in chronic lymphocytic leukemia (CLL): Evidence of in vitro secretion following activation of CLL cells. Leuk Res 2010; 34: e248-9.

13. Hallek M, Langenmayer I, Nerl C, et al. Elevated serum thymidine kinase levels identify a subgroup at high risk of disease progression in early non-smoldering CLL. Blood 1999; 93: 1732-7.

14. Sarfati M, Chevret S, Chastang C, et al. Prognostic importance of serum soluble CD23 level in chronic lymphocytic leukemia. Blood 1996; 88: 4259-64.

15. Montserrat E, Sanchez-Bisono J, Viñolas N, Rozman C. Lymphocyte doubling time in chronic lymphocytic leukaemia: analysis of its prognostic significance. Br J Haematol 1986; 62: 567-75.

16. Rozman C, Montserrat E, Rodríguez-Fernández JM, et al. Bone marrow histologic pattern - the best single prognostic parameter in chronic lymphocytic leukemia: a multivariate survival analysis of 329 cases. Blood 1984; 64: 642-8.

17. Hamblin TJ, Davis Z, Gardiner A, Oscier DG, Stevenson FK. Unmutated Ig VH genes are associated with a more aggressive form of chronic lymphocytic leukemia. Blood 1999; 94: 1848-54.

18. Damle RN, Wasil T, Fais F, et al. Ig V gene mutation status and CD38 expression as a novel prognostic indicators in chronic lymphocytic leukemia. Blood 1999; 94: 1840-7. 
19. Stilgenbauer S, Bullinger L, Lichter P, Döhner H. Genetics of chronic lymphocytic leukemia: genomic aberrations and $\mathrm{V}(\mathrm{H})$ gene mutation status in pathogenesis and clinical course. Leukemia 2002; 16: 993-1007.

20. Crespo M, Bosch F, Villamor N, et al. ZAP-70 expression as a surrogate for immunoglobulin-variable-region mutations in chronic lymphocytic leukaemia. N Engl J Med 2003; 348: 1764-75.

21. Dürig J, Nückel H, Cremer $M$, et al. ZAP-70 expression is a prognostic factor in chronic lymphocytic leukaemia. Leukemia 2003; 17: 2426-34.

22. Hus I, Podhorecka M, Bojarska-Junak A, Roliński J, Schmitt M, Sieklucka M, Wasik-Szczepanek E, Dmoszyńska A. The clinical sig nificance of ZAP-70 and CD38 expression in B-cell chronic lymphocytic leukaemia. Ann Oncol 2006; 17: 683-90.

23. Ibrahim S, Keating M, Do KA, et al. CD38 expression as an important prognostic factor in B-cell chronic lymphocytic leukemia. Blood 2001; 98: 181-6.

24. Hamblin TJ, Orchard JA, Ibbotson RE, Davis Z, Thomas PW, Stevenson FK, Oscier DG. CD38 expression and immunoglobulin variable region mutations are independent prognostic variables in chronic lymphocytic leukemia, but CD38 expression may vary during the course of the disease. Blood 2002; 99: 1023-9.

25. Döhner H, Stilgenbauer S, Benner A, et al. Genomic aberrations and survival in chronic lymphocytic leukemia. N Engl J Med 2000; 343: 1910-6.

26. Döhner H, Stilgenbauer S, James MR, et al. 11q deletions identify a new subset of B-cell chronic lymphocytic leukemia characterized by extensive nodal involvement and inferior prognosis. Blood 1997; 89: 2516-22.

27. Hallek M, Fischer K, Fingerle-Rowson G, et al. International Group of Investigators; German Chronic Lymphocytic Leukaemia Study Group. Addition of rituximab to fludarabine and cyclophosphamide in patients with chronic lymphocytic leukaemia: a ran domised, open-label, phase 3 trial. Lancet 2010; 376: 1164-74.

28. Linn BS, Linn MW, Gurel L. Cumulative illness rating scale. J Am Geriatr Soc 1968; 16: 622-6.

29. Ghielmini M, Vitolo U, Kimby E, et al. Panel Members of the 1st ESMO Consensus Conference on Malignant Lymphoma. ESMO Guidelines consensus conference on malignant lymphoma 2011 part 1: diffuse large B-cell lymphoma (DLBCL), follicular lymphoma (FL) and chronic lymphocytic leukemia (CLL). Ann Oncol 2013; 24: 561-76.

30. Robak T, Jamroziak K, Gora-Tybor J, et al. Comparison of cladribine plus cyclophosphamide with fludarabine plus cyclophosphamide as first-line therapy for chronic lymphocytic leukemia: a phase II randomized study by the Polish Adult Leukemia Group (PALG-CLL3 Study). J Clin Oncol 2010; 28: 1863-9.

31. Hallek M. Therapy of chronic lymphocytic leukaemia. Best Pract Res Clin Haematol 2010; 23: 85-96.

32. Böttcher S, Ritgen M, Fischer K, et al. Minimal residual disease quantification is an independent predictor of progression-free and overall survival in chronic lymphocytic leukemia: a multivariate analysis from the randomized GCLLSG CLL8 trial. J Clin Oncol 2012; 30: 980-8.

33. Eichhorst B, Fink AM, Busch R, et al. Frontline chemoimmunotherapy with fludarabine (F), cyclophosphamide (C), and rituximab (R) (FCR) shows superior efficacy in comparison to bendamustine (B) and rituximab (BR) in previously untreated and physically fit patients (pts) with advanced chronic lymphocytic leukemia (CLL): Final analysis of an international, randomized study of the german CLL study group (GCLLSG) (CLL10 study). Blood 2014; 124: abstract 19.

34. Foà R, Del Giudice I, Cuneo A, et al. Chlorambucil plus rituximab with or without maintenance rituximab as first-line treatment for elderly chronic lymphocytic leukemia patients. Am J Hematol 2014; 89: 480-6.

35. Hillmen P, Gribben JG, Follows GA, et al. Rituximab plus chlorambucil as first line treatment for chronic lymphocytic leukemia (CLL): final analysis of an open-label phase II study. J Clin Oncol 2014, 20: 1236-41.

36. Goede V, Fischer K, Busch R, et al. Obinutuzumab plus chlorambucil in patients with CLL and coexisting conditions. N Engl J Med 2014; 370: 1101-10.
37. Goede V, Fischer K, Engelke A, et al. Obinutuzumab as frontline treatment of chronic lymphocytic leukemia: updated results of the CLL11 study. Leukemia 2015; 29: 1602-4.

38. Hillmen P, Robak T, Janssens A, et al.; COMPLEMENT 1 Study Investigators. Chlorambucil plus ofatumumab versus chlorambucil alone in previously untreated patients with chronic lymphocytic leukaemia (COMPLEMENT 1): a randomised, multicentre, open-label phase 3 trial. Lancet 2015; 385: 1873-83.

39. Eichhorst B, Robak T, Montserrat E, Ghia P, Hillmen P, Hallek M, Buske C; ESMO Guidelines Committee. Chronic lymphocytic leukaemia: ESMO Clinical Practice Guidelines for diagnosis, treatment and follow-up. Ann Oncol. 2015; 26 Suppl 5: v78-v84.

40. Zelenetz AD, Gordon LI, Wierda WG, et al. Chronic lymphocytic leukemia/small lymphocytic lymphoma, version 1.2015. J Natl Compr Canc Netw 2015; 13: 326-62.

41. Knauf WU, Lissichkov T, Aldaoud A, et al. Phase III randomized study of bendamustine compared with chlorambucil in previously untreated patients with chronic lymphocytic leukemia. J Clin Oncol 2009; 27: 4378-84

42. Knauf WU, Lissitchkov T, Aldaoud A, et al. Bendamustine compared with chlorambucil in previously untreated patients with chronic lymphocytic leukaemia: updated results of a randomized phase III trial. Br J Haematol 2012; 159: 67-77.

43. Fischer K, Cramer P, Busch R, et al. Bendamustine in combination with rituximab for previously untreated patients with chronic lymphocytic leukemia: a multicenter phase II trial of the German Chronic Lymphocytic Leukemia Study Group. J Clin Oncol 2012; 30: 3209-16.

44. Foon KA, Boyiadzis M, Land SR, et al. Chemoimmunotherapy with low-dose fludarabine and cyclophosphamide and high dose rituximab in previously untreated patients with chronic lymphocytic leukemia. J Clin Oncol 2009; 27: 498-503.

45. Smolej L, Spacek M, Brychtova Y, et al. Low-dose fludarabine and cyclophosphamide combined with reituximab in the treatment of elderly/comorbind patients with chronic lymphocytic leukemia/ small lymphocytic lymphoma (CLL/SLL): preliminary results of Project Q-Lite by Czech Study Group. Blood 2010; 116: abstract 2466.

46. Forconi F, Fabbri A, Lenoci M, Sozzi E, Gozzetti A, Tassi M, Raspadori D, Lauria F. Low-dose oral fludarabine plus cyclophosphamide in elderly patients with untreated and relapsed or refractory chronic lymphocytic leukaemia. Hematol Oncol 2008; 26: 247-51.

47. Robak T, Dmoszynska A, Solal-Céligny P, et al. Rituximab plus fludarabine and cyclophosphamide prolongs progression-free survival compared with fludarabine andcyclophosphamide alone in previously treated chronic lymphocytic leukemia. J Clin Oncol 2010; 28: 1756-65.

48. Byrd JC, Furman RR, Coutre SE, et al. Targeting BTK with ibrutinib in relapsed chronic lymphocytic leukemia. N Engl J Med 2013; 369: 32-42.

49. Furman RR, Sharman JP, Coutre SE, et al. Idelalisib and rituximab in relapsed chronic lymphocytic leukemia. N Engl J Med 2014; 370: 997-1007.

50. Fischer K, Cramer P, Busch R, et al. Bendamustine combined with rituximab in patients with relapsed and/or refractory chronic lymphocytic leukemia: a multicenter phase II trial of the German Chronic Lymphocytic Leukemia Study Group. J Clin Oncol 2011; 29: 3559-66.

51. Castro JE, Sandoval-Sus JD, Bole J, Rassenti L, Kipps TJ. Rituximab in combination with high-dose methylprednisolone for the treatment of fludarabine refractory high-risk chronic lymphocytic leukemia. Leukemia 2008; 22: 2048-53.

52. Rossignol J, Michallet AS, Oberic et al. Rituximab-cyclophosphamide-dexamethasone combination in the management of autoimmune cytopenias associated with chronic lymphocytic leukemia. Leukemia 2011; 25: 473-8

53. Michallet AS, Rossignol J, Cazin B, Ysebaert L. Rituximab-cyclophosphamide-dexamethasone combination in management of autoimmune cytopenias associated with chronic lymphocytic leukemia. Leuk Lymphoma 2011; 52: 1401-3. 
54. Pettitt AR, Jackson R, Carruthers S, et al. Alemtuzumab in combination with methylprednisolone is a highly effective induction regimen for patients with chronic lymphocytic leukemia and deletion of TP53: final results of the national cancer research institute CLL206 trial. J Clin Oncol 2012; 30: 1647-55.

55. Elter T, Borchmann P, Schulz $\mathrm{H}$, et al. Fludarabine in combination with alemtuzumab is effective and feasible in patients with relapsed or refractory B-cell chronic lymphocytic leukemia: results of a phase II trial. J Clin Oncol 2005; 23: 7024-31.

56. Pileckyte R, Jurgutis $M$, Valceckiene $V$, et al. Dose-dense high-dose methylprednisolone and rituximab in the treatment of relapsed or refractory high-risk chronic lymphocytic leukemia. Leuk Lymphoma 2011; 52: 1055-65.

57. Xu W, Miao KR, Hong M, et al. High-dose methylprednisolone can induce remissions in patients with fludarabine-refractory chronic lymphocytic leukaemia. Eur J Cancer 2010; 46: 2145-9.

58. Seymour JF, Davids MS, Pagel JM, et al. Updated results of a phase I first-in-human study of the BCL-2 inhibitor ABT-199 (GDC-0199) in patients with relapsed/refractory (R/R) chronic lymphocytic leukemia (CLL). J Clin Oncol 2013; 31: Abstr 7018.

\section{Address for correspondence}

\section{Prof. Iwona Hus}

Department of Clinical Transplantology

Medical University of Lublin, Poland

e-mail: iwonach.hus@gmail.com

Submitted: 30.09 .2014

Accepted: 8.10 .2014 